\title{
FUNDAMENTAL INTERACTIONS OF NATURE: CLASSICAL UNIFICATION OF GRAVITATIONAL AND ELECTROMAGNETIC FORCES
}

\author{
Alfred Mishi", Charity Kuje, Arigu Sabari, John Ojosipe, Deborah Adigun, \\ Chinedu Egbogu \& Daniel Dusu
}

Department of Physics, Faculty of Natural Sciences, University of Jos.

*Corresponding Author: fredsteingenius@gmail.com

\begin{abstract}
We have looked at the four fundamental interactions, the interaction between imaginary energies. This been the electromagnetic (between electric charges), weak (between electric and colour charges), and strong (between colour charges) interactions. Among the four fundamental elements, there are ten (six real and four imaginary) fundamental interactions. We also have presented a Classical theory of unification of gravitational force and the electromagnetic force based on generalization of Newton's law of gravitation to include a dynamic term inferred from the Lorentz force of electromagnetic interaction. This dynamic term alone in the gravitational force is enough to develop the entire dynamic theory of gravitation parallel to that of electrodynamics. This theory naturally solves the mystery of action-at-distance. It has been shown that the inverse square law of the static and the dynamic forces is the result of the conservation of mass (Gauss's Law) and the total momentum (Wang's Law). The Wang's Law been a new discovery. The new theory also predicts that the gravitational force is transmitted through propagation of gravitational waves at the speed of light.
\end{abstract}

KEYWORDS: Classical Unification of Gravitational, Electromagnetic Forces, Strong Force, Weak Force, Imaginary Force

\section{INTRODUCTION}

Classical elements typically refer to the concepts of earth, water, air, fire, and aether, which were proposed to explain the nature and complexity of all matter in terms of simpler substances [1]. Ancient cultures in Greece, Persia, Babylonia, Japan, Tibet, and India had all similar lists, sometimes referring in local languages to "air" as "wind" and the fifth element as "void" [2]. In traditional Chinese $\mathrm{Wu}$ Xing (or five-element) theory, the space and wind are replaced by metal and wood. All the natural phenomena are described by the interactions of the five elements. There are two cycles of balances: generating (or sheng in Chinese) and overcoming (or ke in Chinese) cycles. The generating cycle includes that wood feeds fire, fire creates earth (or ash), earth bears metal, metal carries water, and water nourish wood; while the overcoming cycle includes that wood parts earth, earth absorbs water, water quenches fire, fire melts metal, and metal chops wood. According to the modern scientific view, how many elements does the nature have? How do these fundamental elements interact with each other? It is well known that there have been four fundamental interactions found in the nature. They are the gravitational, electromagnetic, weak, and strong interactions. The gravitational interaction is an interaction between masses. The electromagnetic interaction is an interaction between electric charges. The strong interaction is an interaction between colour charges. What is the 
weak interaction? Elementary particles are usually classified into two categories: hadrons and leptons. Hadrons participate in both strong and weak interactions, but leptons can only participate in the weak interaction. If the weak interaction is an interaction between weak charges, what is the weak charge? How many types of weak charges? Are the weak charges in hadrons different from those in leptons? Do we really need weak charges for the weak interaction? All of these are still unclear although the weak interaction has been extensively investigated for many decades. Some studies of particular particles show that the weak charges might be proportional to electric charges [3].

In this paper, we look at the nature of fundamental interactions and a Classical Unification of Gravitational and Electromagnetic Forces. The nature fundamental interactions are the forces among these fundamental elements. The weak interaction is considered as an interaction between colour charges and electric charges [3].

All the fundamental interactions can be classically unified into a single interaction between complex energies. The interaction between real energies is gravitational interaction. By including the massless radiation, we have three types of gravitational forces. The interaction between imaginary energies are electromagnetic (between electric charges), weak (between electric and colour charges), and strong (between colour charges) interactions. In addition, we have four types of imaginary forces (between real and imaginary energies): mass-electric charge interaction, radiation-electric charge interaction, mass-colour charge interaction, and radiation-colour charge interaction. Among the four fundamental elements, we have in total ten (six real and four imaginary) fundamental interactions [3].

\section{Fundamental Interactions of Nature}

Fundamental interactions of nature are all possible interactions between the four fundamental elements of nature. Each of the four fundamental elements is a form of energy (either real or imaginary), the fundamental interactions can be unified as a single interaction between complex energies given by [3]

$$
\vec{F}_{E E}=-G \frac{E_{1} E_{2}}{c^{4} r^{2}} \hat{\vec{r}}
$$

where $E_{1}$ and $E_{2}$ are the complex energy given by

$$
\begin{aligned}
& E_{1}=E_{1}^{M}+E_{1}^{\gamma}+i\left(E_{1}^{Q}+E_{1}^{C}\right) \\
& E_{2}=E_{2}^{M}+E_{2}^{\gamma}+i\left(E_{2}^{Q}+E_{2}^{C}\right)
\end{aligned}
$$

Replacing $E_{1}$ and $E_{2}$ by using the energy expression 2 and 3, we have that

$$
\vec{F}_{E E}=\vec{F}_{R R}+\vec{F}_{I I}+i \vec{F}_{R I}
$$




$$
\begin{gathered}
=-G \frac{M_{1} M_{2}}{r^{2}} \hat{\vec{r}}-G \frac{M_{1} h v_{2}+M_{2} h v_{1}}{c^{2} r^{2}} \hat{\vec{r}}-G \frac{h v_{1} h v_{2}}{c^{4} r^{2}} \hat{\vec{r}}+\frac{Q_{1} Q_{2}}{r^{2}} \hat{\vec{r}} \\
+\frac{Q_{1} C_{2}+Q_{2} C_{1}}{r^{2}} \hat{\vec{r}}+\frac{C_{1} C_{2}}{r^{2}} \hat{\vec{r}} \\
-i \sqrt{G} \frac{M_{1} Q_{2}+M_{2} Q_{1}}{r^{2}} \hat{\vec{r}}-i \sqrt{G} \frac{M_{1} C_{2}+M_{2} C_{1}}{r^{2}} \hat{\vec{r}}-i \sqrt{G} \frac{h v_{1} Q_{2}+h v_{2} Q_{1}}{c^{2} r^{2}} \hat{\vec{r}} \\
-i \sqrt{G} \frac{h v_{1} C_{2}+h v_{2} C_{1}}{c^{2} r^{2}} \hat{\vec{r}} \\
\equiv \vec{F}_{M M}+\vec{F}_{M \gamma}+\vec{F}_{\gamma \gamma}+\vec{F}_{Q Q}+\vec{F}_{Q C}+\vec{F}_{C C}+i \vec{F}_{M Q}+i \vec{F}_{M C}+i \vec{F}_{Q \gamma}+i \vec{F}_{C \gamma}
\end{gathered}
$$

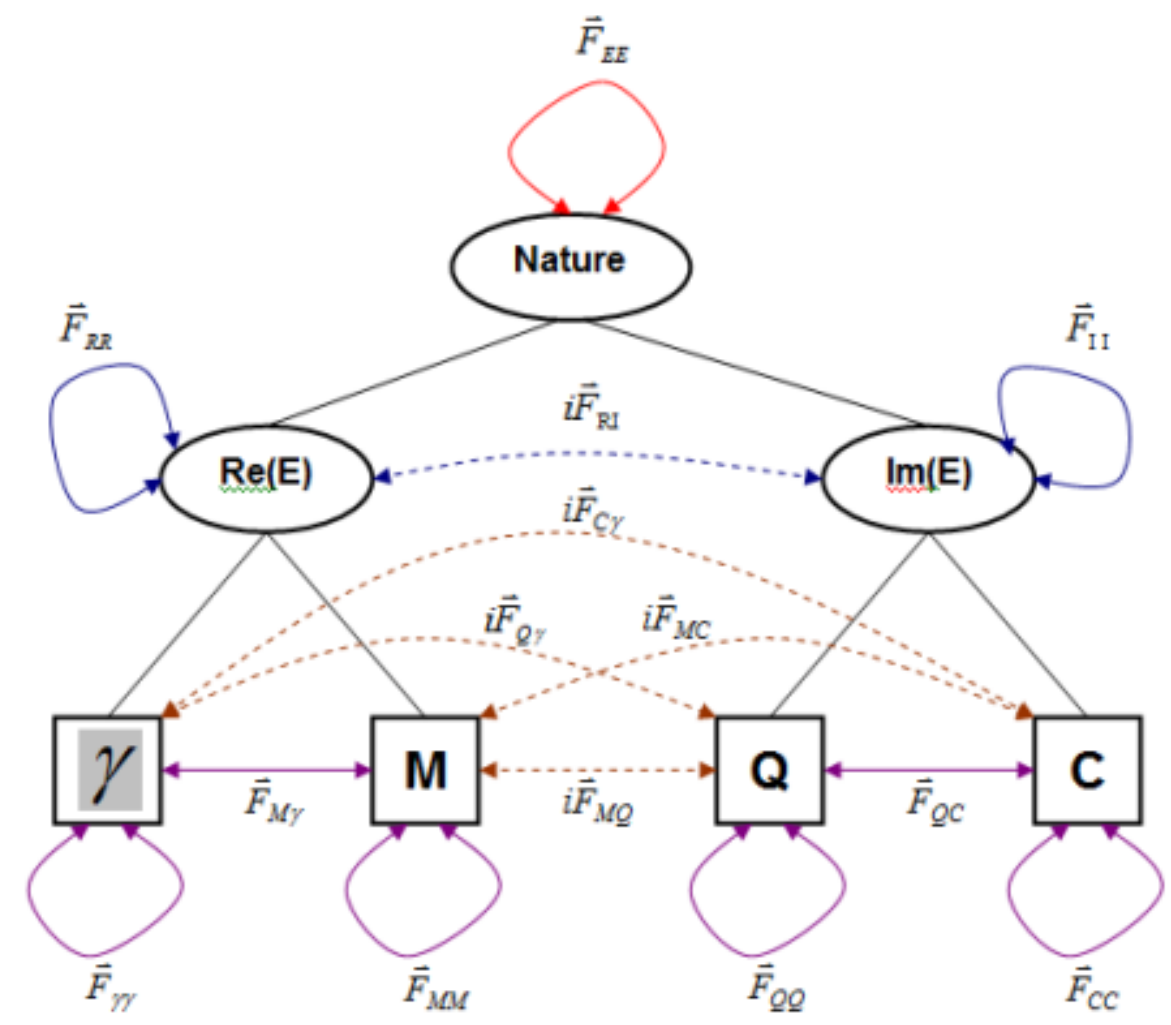

Figure 1: Fundamental Interactions among four Fundamental Elements of Nature: Mass, Radiation, Electric Charge and Colour Charge. 
Mass and radiation are real energies, while electric and colour charges are imaginary energies. The nature is a system of complex energy and all the fundamental interactions of nature are classically unified into a single interaction between complex energies. There are six real and four imaginary interactions among the four fundamental elements [3].

It is seen that the interaction between complex energies $\vec{F}_{E E}$ is decoupled into the real-real energy interaction $\vec{F}_{R R}$, the imaginary-imaginary energy interaction $\vec{F}_{I I}$, and the real-imaginary energy interaction $i \vec{F}_{R I}$. The real-real energy interaction $\vec{F}_{R R}$ is decoupled into the mass-mass interaction $\vec{F}_{M M}$, the radiation-radiation interaction $\vec{F}_{\gamma \gamma}$, and the mass-radiation interaction $\vec{F}_{M \gamma}$. The imaginary-imaginary energy interaction $\vec{F}_{I I}$ is decoupled into the interaction between electric charges $\vec{F}_{Q Q}$, the interaction between color charges $\vec{F}_{C C}$, and the interaction between electric and color charges $\vec{F}_{Q C}$. The real-imaginary energy interaction $i \vec{F}_{R I}$ is decoupled into the mass-electric charge interaction $i \vec{F}_{M Q}$, the mass-color charge interaction $i \vec{F}_{M C}$, the radiation-electric charge interaction $i \vec{F}_{Q \gamma}$, the radiation-colour charge interaction $i \vec{F}_{C \gamma}$. All these interactions as shown in Eq. (4) can be represented by Figure 1 above.

\section{Gravitational Force}

The force $\vec{F}_{M M}$ represents Newton's law for the gravitational interaction between two masses. This force governs the orbital motion of the solar system. The force $\vec{F}_{M \gamma}$ is the gravitational interaction between mass and radiation. The force $\vec{F}_{\gamma \gamma}$ is the gravitational interaction between radiation and radiation. These three types of gravitational interactions are categorized from the interaction between real energies. Calculating the work done by this mass-radiation force on a photon, we can derive the Einsteinian gravitational redshift without using the Einsteinian general relativity [3]

$$
Z_{G}=\frac{\lambda_{o}-\lambda_{e}}{\lambda_{e}}=e^{\left(\frac{G M}{c^{2} R}\right)-1}
$$

In the weak field approximation, it reduces

$$
Z_{G} \simeq \frac{G M}{c^{2} R}
$$

Similarly, calculating the work done on a photon from an object by the radiation-radiation gravitation $\vec{F}_{\gamma \gamma}$, we get a radiation redshift,

$$
Z_{\gamma}=\frac{4 G M}{15 c^{5}} \sigma A T_{c}^{4}+\frac{G}{c^{5}} \sigma A T_{s}^{4}
$$

where $\sigma$ is the Stephan-Boltzmann constant, $\mathrm{A}$ is the surface area, $T_{c}$ is the temperature at the centre, $T_{s}$ is the temperature on the surface. Here we have assumed that the inside temperature 
linearly decreases from the centre to the surface. The radiation redshift contains two parts. The first term is contributed by the inside radiation. The other is contributed by the outside radiation. The redshift contributed by the outside radiation is negligible because $T_{s} \ll T_{c} \quad[3]$.

The radiation redshift derived here is significantly small in comparison with the empirical expression of radiation redshift proposed by Finlay-Freundlich. For the Sun with $T_{c}=$ $1.5 \times 10^{7} \mathrm{~K}$ and $T_{s}=6 \times 10^{3} \mathrm{~K}$, the radiation redshift is only about $Z_{\gamma}=1.3 \times 10^{-13}$, which is much smaller than the gravitational redshift $Z_{G}=2.1 \times 10^{-6}[3]$.

\section{Electromagnetic Force}

The force $\vec{F}_{Q Q}$ represents Coulomb's law for the electromagnetic interaction between two electric charges. Electric charges have two varieties and thus three types of interactions [3]:

1) repelling between positive electric charges $\vec{F}_{++}$,

Fig. 2: Six types of strong interactions between colour charges: red-red, green-green, blue-blue, red-green, red-blue, and green-blue interactions.

2) repelling between negative electric charges $\vec{F}_{--}$, and

3) attracting between positive and negative electric charges $\vec{F}_{+-}$

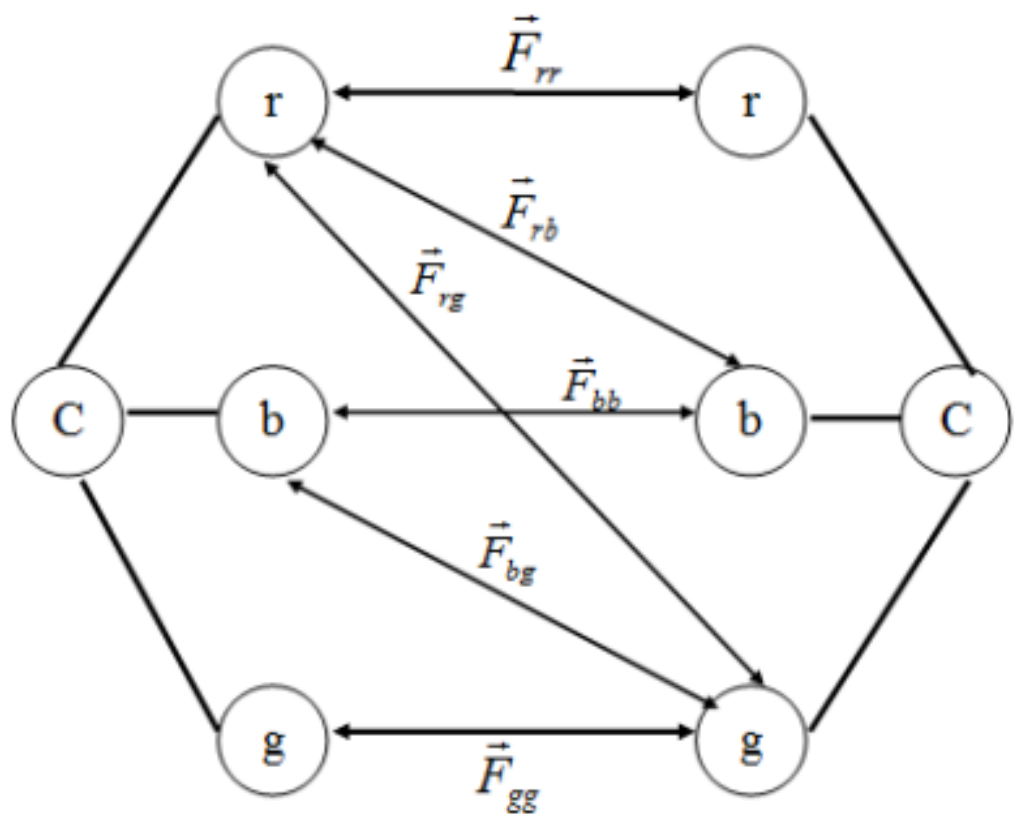

Figure 2: Six Types of Strong Interactions Between Colour Charges: Red-Red, GreenGreen, Blue-Blue, Red-Green, red-Blue, and Green-Blue Interactions [3].

Figure 2 shows the three types of Coulomb interactions between two electric charges. 


\section{Strong Force}

The force $\vec{F}_{C C}$ is the strong interaction between colour and colour charges. Colour charges have three varieties: red, blue, and green and thus six types of interactions [3]:

1) the red-red interaction $\vec{F}_{r r}$,

2) the blue-blue interaction $\vec{F}_{b b}$,

3) the green-green interaction $\vec{F}_{g g}$,

4) the red-blue interaction $\vec{F}_{r b}$,

5) the red-green interaction $\vec{F}_{r g}$, and

6) the blue-green interaction $\vec{F}_{b g}$.

Figure 2 shows these six types of colour interactions.

Considering the strong interaction to be asymptotically free, we replace the colour charge by [3]

$$
C \rightarrow r C
$$

this assumption represents that the colour charge becomes less colourful if it is closer to each other, i.e., asymptotically colourless. Then the strong interaction between colour charges can be rewritten by

$$
\vec{F}_{C C}=C_{1} C_{2} \hat{\vec{r}}
$$

which is independent of the radial distance and consistent with measurement.

The strong interaction is the only one that can change the colour of quarks in a hadron. A typical strong interaction is proton-neutron scattering, $p+n \rightarrow n+p$. This is an interaction between the colour charge of one up quark in proton and the colour charge of one down quark in neutron via exchanging a $\pi^{+}, u+d \rightarrow d+u$. In other words, during this proton-neutron scattering an up quark in the proton changes into a down quark by emitting a $\pi^{+}$, meanwhile a down quark in the neutron changes into an up quark by absorbing the $\pi^{+}$. Another typical strong interaction is delta decay, $\Delta^{0} \rightarrow p+\pi^{-}$. This is an interaction between the colour charge of one down quark and the colour charges of the other two quarks. In this interaction, a down quark emits a $\pi^{-}$and then becomes a up quark, $d \rightarrow u+\pi^{-}$[3]. 


\section{Weak Force}

The force $\vec{F}_{Q C}$ is the weak interaction between electric and colour charges. Considering electric charges with two varieties (positive and negative) and colour charges with three varieties (red, blue, and green), we have also six types of weak interaction [3]:

1) the positive-red interaction $\vec{F}_{+r}$,

2) the positive-blue interaction $\vec{F}_{+b}$,

3) the positive-green interaction $\vec{F}_{+g}$,

4) the negative-red interaction $\vec{F}_{-r}$,

5) the negative-blue interaction $\vec{F}_{-b}$, and

6) the negative-green interaction $\vec{F}_{-g}$.

Figure 3 shows these six types of electric-colour charge interactions [3].

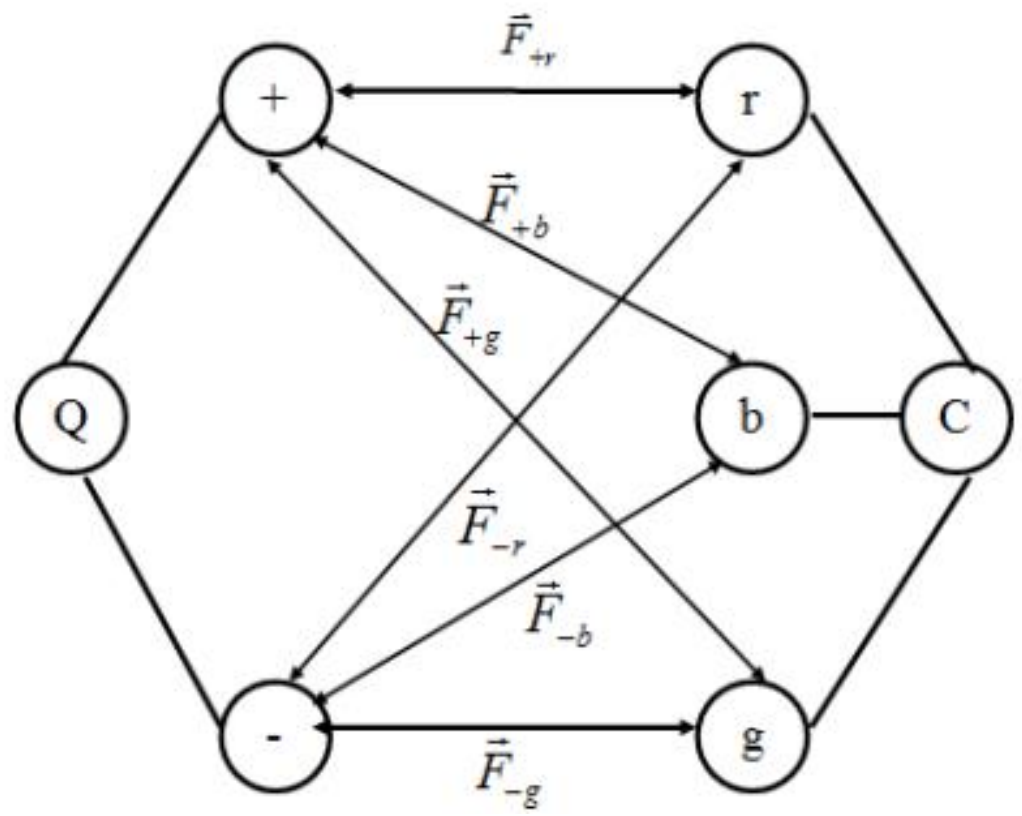

Figure 3: Six Types of Weak Interactions Between Electric and Colour Charges: Positive-Red, Positive-Green, Positive-Blue, Negative-Red, Negative-Green, And Negative-Blue Interactions.

Considering equation (8), we can represent the weak interaction by [3]

$$
\vec{F}_{Q C}=\frac{Q C}{r} \hat{\vec{r}}
$$

which is inversely proportional to the radial distance and consistent with measurement. 
The weak interaction is the only one that can change the flavours of quarks in a hadron. A typical weak interaction is the neutron decay, $n \rightarrow p+e^{-}+\bar{v}_{e}$. In this process, a down quark in the neutron changes into an up quark by emitting $W^{-}$boson, which lives about $10^{-26}$ seconds and then breaks into a high-energy electron and an electron antineutrino, i.e., $d \rightarrow$ $u+W^{-}$and then $W^{-} \rightarrow u+e^{-}+\bar{v}_{e}$. There are actually two interactions involved in this neutron decay. One is the interaction between electric and colour charges inside the down quark, which is changed into an up quark by emitting a $\mathrm{W}$ - boson. Another is the interaction inside $W^{-}$, which is broken into an electron and an electron antineutrino. Since $W^{-}$is composed of an up antiquark and a down quark $(\bar{u} d)$, we suggest that the down quark changes into an up quark by emitting an electron and then the up antiquark and the up quark annihilate into an electron antineutrino. It should be noted that an upper antiquark and an up quark usually forms an $\eta$ particle, which may live about a few tens of nanoseconds and decay into other particles such as photons and pions, which further decay to nuons and nuon neutrinos and antineutrinos. The formation of $\eta$ and decay to photons and pions may explain the solar neutrino missing problem and neutrino oscillations [3].

\section{Imaginary Force}

The other terms with the imaginary number in Eq. (4) are imaginary forces between real and imaginary energies. These imaginary forces should play essential roles in combining or separating imaginary energies with or from real energies. The physics of imaginary forces needs further investigations [3].

\section{Classical Unification of Gravitation and Electromagnetic Forces}

It has been a dream of physicists to unify all the fundamental forces over at least a century. This dream has so far advanced to the first stage of unification of the electromagnetic and the weak forces in the Standard Model of electroweak theory. The next stage of the dream is to include the strong force into this unification - known as the Grand Unification Theory (GUT), which requires an unbelievably high energy in the order of $10^{16} \mathrm{GeV}$ and an accelerator larger than the solar system. It is apparently beyond our reach in the foreseeable future. The last stage of this dream is the Theory of Everything (TOE) to unify all the four forces, including the gravitational force, but the gravitational force seems to be the most persistent and the best fortified castle against unification by particle physicists within the framework of the standard model. Speculative theories have been attempted, with more problems created than solved. Not only it requires an even higher energy - known as the Plank energy scale, about 1000 times higher than the GUT energy scale, but also there are fundamental incompatibilities between the gravitational force and the other fundamental forces. It has been realized that general relativity is incompatible with quantum mechanics [4]. The approach of describing gravity in the framework of quantum field theory $[5,6]$ fails at short distances of the order of the Planck length [4].

Instead of trying to unify all forces, we have looked at a theory to unify the gravitational force with the electromagnetic force strictly within the classical frame work [7]. Our theory is inspired by two observations: (1) Newton's law of gravitation is strikingly similar to Coulomb's law; (2) Newton's law differs from Coulomb's law, equally striking, by lacking a dynamic term dependent on the velocity of the gravitating source. As a result, Newton's law is essentially a static theory unable to describe the propagation of the interaction. This is the theoretical origin of the historical problem of action-at-distance [12]. 
Natural questions arise: Is the lacking of a dynamic term in Newton's law a true manifestation of the law of Nature, or a theoretical miss due to the weakness of the dynamic part of the gravitational force that escaped the detection by observational astronomers and experimental physicists? If the latter is the case, what kind of dynamic term should be added to Newton's law? [12]

Let us try to answer the first question by examining the electromagnetic force, known as the Lorentz force [12]:

$$
\mathbf{F}=k_{1} \frac{q q^{\prime}}{r^{2}}\left[\hat{r}+\frac{1}{c^{2}} \mathrm{v}^{\prime} \times(\mathrm{v} \times \hat{r})\right]
$$

where $k_{1}$ is the Coulomb constant, $\mathrm{c}$ the speed of light, $\mathrm{q}$ and $\mathrm{q}^{\prime}$ the charges, $\mathrm{r}$ the distance between them, $\mathrm{v}$ and $\mathrm{v}^{\prime}$ are the velocities of the two charges, respectively. In the most favourable case, the second term (dynamic term) is weaker than the first term (static term) by a factor of $\left(v v^{\prime} / c^{2}\right)$. Should the gravitational force also include a similar dynamic term, it will be weaker than the static term by the same factor. The orbital velocity $\mathrm{v}^{\prime}$ of the earth is about $30 \mathrm{~km} / \mathrm{s}$ and the velocity $\mathrm{v}$ of the sun about the centre of mass of the solar system is about 0.1 $\mathrm{m} / \mathrm{s}$. These values of the velocities would give a value of $\left(v v^{\prime} / c^{2}\right)$ in the order of $10^{-13}$. That is to say, the dynamic term, if exists, would cause a difference of less than a factor of $10^{-13}$ in the gravitational force between the sun and the planets. It is definitely beyond the ability of the observational astronomers to detect. Although the dynamic term has little effect on the orbital movements of the planets, it is a necessity to describe the propagation of the gravitational wave and answer the question of action-at-distance [12]

If we are to add a dynamic term to Newton's law of gravity, what kind of form would such dynamic term take? Well, it is all too natural to speculate that the dynamic term should take the same form of the Lorentz force considering the striking similarity between Newton's law of gravitation and Coulomb's law [12].

Historically, a gravitational equivalent of the magnetic force has been speculated by Lorrain and Corson in 1970 [8], making a statement that such force "could only be attractive" without any quantitative analysis. Salisbury and Menzel also speculated the similar force with a brief analysis based on a particular example of two stationary mass/charges whose gravitational attraction exactly balances their electrostatic repulsion [9]. Salisbury and Menzel's result based on this special arrangement contradicted the conclusion by Lorrain and Corson and by Sciama [10]. These earlier speculations show that the generalization of Newton's law of gravitation to include a dynamic term is a very natural and logical attempt to develop a more general theory of gravitation. They did not go much farther than just adding a term to Newton's formula. It demonstrated the tremendous challenges facing physicists to develop a logically consistent and systematic general theory of gravitation with mathematical rigor and completeness [12].

In the following sections, we will present a generalized theory of gravitation by adding a dynamic term similar to the Lorentz force. It turns out that the inclusion of a dynamic Lorentz force alone is enough to develop the whole theory of dynamic gravitation and the wave equations. It has been shown that the inverse square law is a result of Gauss' Law and Wang's 
Law. The newly discovered Wang's Law shows that the total linear momentum propagated into space is conserved [12].

\section{Generalization of Newton's Law}

We generalize Newton's Law of gravitation to include a dynamic term similar to the Lorentz force in $\mathrm{Eq}(11)$ [12]:

$$
\mathbf{F}=-G \frac{m m^{\prime}}{r^{2}}\left[\hat{r}+\frac{1}{c^{2}} \mathrm{v}^{\prime} \times(\mathrm{v} \times \hat{r})\right]
$$

where $\mathrm{v}$ and $\mathrm{v}^{\prime}$ are the velocities of the masses $\mathrm{m}$ and $\mathrm{m}^{\prime}$, respectively.

The first term of the gravitational force is static [12]:

$$
\mathbf{F}_{1}=-G \frac{m m^{\prime}}{r^{2}} \hat{r}
$$

We can define a static field [12]:

$$
g=\frac{\mathbf{F}_{1}}{m^{\prime}}=-G \frac{m}{r^{2}} \hat{r}
$$

The second term can be written as [12].

$$
\begin{gathered}
\mathbf{F}_{2}=-G \frac{m m^{\prime}}{r^{2}} \frac{1}{c^{2}} \mathrm{v}^{\prime} \times(\mathrm{v} \times \hat{r}) \\
=-G \frac{m m^{\prime}}{r^{2}}\left[(\boldsymbol{\beta} \cdot \hat{r}) \boldsymbol{\beta}-\left(\boldsymbol{\beta}^{\prime} \cdot \boldsymbol{\beta}\right) \hat{r}\right]=\mathbf{M} \cdot m^{\prime} \boldsymbol{\beta}^{\prime}
\end{gathered}
$$

where $\boldsymbol{\beta}$ and $\boldsymbol{\beta}^{\prime}$ are the ratios of velocities over the speed of light, $\mathbf{M}$ is a second rank antisymmetric tensor constructed by the usual rule of dyadic of two vectors [12]:

$$
\mathbf{M}=\frac{G m}{c r^{3}}(\vec{r} \mathrm{v}-\mathrm{v} \vec{r})=\frac{G m}{c r^{3}}\left(\begin{array}{ccc}
0 & v_{y} x-v_{x} y & v_{z} x-v_{x} z \\
v_{x} y-v_{y} x & 0 & v_{z} y-v_{y} z \\
v_{x} z-v_{z} x & v_{y} z-v_{z} y & 0
\end{array}\right)
$$


Since the angular momentum

$$
\mathbf{L}=\mathbf{r} \times \mathbf{p}=m \mathbf{r} \times \mathbf{v}
$$

we have

$$
\mathbf{M}=\frac{G}{c r^{3}}\left(\begin{array}{ccc}
0 & L_{z} & -L_{y} \\
-L_{z} & 0 & L_{x} \\
L_{y} & -L_{x} & 0
\end{array}\right)
$$

Define a vector $\mathbf{d}[12]$ :

$$
\mathbf{d}=\frac{G}{c r^{3}} \mathbf{L}=\frac{G}{c r^{2}} \hat{r} \times \mathrm{v}
$$

We have

$$
M=\left(\begin{array}{ccc}
0 & d_{z} & -d_{y} \\
-d_{z} & 0 & d_{x} \\
d_{y} & -d_{x} & 0
\end{array}\right)
$$

Apparently, the vector $\mathbf{d}$ is proportional to the angular momentum $\mathbf{L}$ of the moving mass about the point of interest and inversely proportional to the cube of the distance $r$. Since $\mathbf{L}$ is proportional to r, Eqs. (18) and (19) manifest inverse square law of $\mathbf{d}$ and $\mathbf{M}$. We will call $\mathbf{d}$ the dynamic field, and $\mathbf{M}$ the dynamic field tensor [12].

The gravitational force can be expressed as [12]

$$
\mathbf{F}=m^{\prime}\left[\mathbf{g}+\mathbf{M} \cdot \boldsymbol{\beta}^{\prime}\right]=m^{\prime}\left[\mathbf{g}+\boldsymbol{\beta}^{\prime} \times \mathbf{d}\right]
$$

The total gravitational field $\mathrm{f}$ is defined as the total gravitational force per unit mass [12]:

$$
\mathbf{f}=\mathbf{g}+\mathbf{M} \cdot \boldsymbol{\beta}^{\prime}=\mathbf{g}+\boldsymbol{\beta}^{\prime} \times \mathbf{d}
$$




\section{Gauss' Law and Wang's Law}

It can be shown [7] that

$$
\nabla \cdot g=-4 \pi G \rho
$$

where

$$
\rho=\frac{d m}{d V}
$$

is the local mass density, $\mathrm{V}$ being the volume

The similar closed surface integral of the dynamic field tensor M is [7]

$$
\oiint \mathbf{M} \cdot d \boldsymbol{\sigma}=-\frac{8 \pi G m}{3 c}\left(\begin{array}{l}
v_{x} \\
v_{y} \\
v_{z}
\end{array}\right)=-\frac{8 \pi G}{3 c} m \mathrm{v}=-\frac{8 \pi G}{3 c} \mathbf{p}
$$

where $\mathbf{p}=\mathrm{mv}$ is the linear momentum of the gravitating mass. Eq. (23) is Gauss' law. Eq. (25) is referred to as Wang's Law [4], which says that the closed integration of the dynamic field tensor $\mathbf{M}$ is a constant proportional to the linear momentum of the moving mass. Wang's law is therefore a statement that the total linear momentum transmitted through the gravitational field into the space is conserved. The constant $-4 \pi G m$ in Gauss' law is called the total static flux. The constant $-\frac{8 \pi G}{3 c} m \mathrm{v}$ is called the total dynamic flux. We can then speak of conservation of the total static and dynamic fluxes of the gravitational field.

The conservation of the total static flux and the dynamic flux reveals how the gravitational interaction is transmitted. A mass $m$ at rest in space causes stress to the medium ether. If the mass is at rest the total stress flux is equal to $-4 \pi G m$. If the mass is moving with velocity $\mathbf{v}$, it will cause additional dynamic stress to the ether. The momentum-induced stress will then propagate into the space, with the total linear momentum constant and distributed over the total solid angle. The static and the dynamic fields are simply the stress fluxes per unit area and therefore inversely proportional to the distance squared. It naturally explains the inverse square law of the electromagnetic and the gravitational forces [12].

The significance of this discovery can be appreciated if we recall the history of the discovery of inverse square law. Newton discovered that in order to explain Kepler's third law, his newly proposed law of gravitation must be inversely proportional to the square of the distance. The power index 2 was pretty much a result of fitting the data since Kepler's laws ware all results of empirical observation. People can always ask if the index is exactly equal to two or nearly equal to two. As a matter of fact, some theoretical textbooks actually give a derivation of the inverse square law along this line. Coulomb's law and Biot-Savart law are also empirical laws. Empirical laws are always subject to experimental errors. The issue is finally settled by Gauss' 
law and Wang's law. The inverse square law is exact as long as the surface area of a sphere is exactly equal to the radius squared [12].

\section{The Wave Equation of a Gravitational Field}

It can be shown that [7]

$$
\begin{gathered}
\nabla . \mathbf{f}=-4 \pi G\left(\rho+\frac{2}{3 c} \boldsymbol{\beta}^{\prime} \cdot \mathbf{j}\right) \\
\nabla \cdot \mathbf{d}=0 \\
\nabla \times \mathbf{d}=\frac{1}{c} \frac{\partial g}{\partial t}+\frac{8 \pi G}{3 c} \mathbf{j} \\
\nabla \times \mathbf{f}=-\frac{1}{c} \frac{\partial \mathbf{d}}{\partial t}
\end{gathered}
$$

where

$$
\mathbf{j}=\rho \mathrm{v}=\frac{\mathbf{p}}{V}
$$

$\mathbf{j}$ is the momentum density, i.e., the momentum per unit volume. $\mathbf{p}$ is the total momentum of the mass contained in the volume $\mathrm{V}$. $\mathbf{j}$ is also the current density (current per unit area).

Eqs. (26) -(29) constitute a set of fundamental equations of the gravitational field. In a free space where $\rho=0$ and, $\mathrm{j}=0$, these equations take simple and symmetric form [12]:

$$
\begin{gathered}
\nabla \cdot \mathbf{f}=0 \\
\nabla \cdot \mathbf{d}=0 \\
\nabla \times \mathbf{d}=\frac{1}{c} \frac{\partial \mathbf{f}}{\partial t}
\end{gathered}
$$




$$
\nabla \times \mathbf{f}=-\frac{1}{c} \frac{\partial \mathbf{d}}{\partial t}
$$

Eqs. (31) -(34) form a complete set of equations that describe the propagation of the gravitational wave in vacuum. Routine manipulation of this set of equations yields a wave equation [12]:

$$
\nabla^{2} \mathrm{f}-\frac{1}{c^{2}} \frac{\partial^{2} \mathrm{f}}{\partial t^{2}}=0
$$

with solution

$$
\mathbf{f}=\mathbf{f}_{0} \sin (\omega t-\mathbf{k} \cdot \mathbf{r})
$$

where

$$
\omega=2 \pi \mathrm{v}=\frac{2 \pi}{T}
$$

is the angular frequency. $v$ and $\mathrm{T}$ are the frequency and the period. $\mathbf{k}=k \hat{\mathbf{k}}$ is the wave number:

$$
k=\frac{2 \pi}{\lambda}
$$

And

$$
\lambda=c T
$$

is the wavelength. $\mathrm{c}$ is the speed of light.

A similar procedure yields a wave function for $\mathbf{d}$ [12]:

$$
\mathbf{d}=\mathbf{d}_{0} \sin (\omega t-\mathbf{k} \cdot \mathbf{r})
$$




\section{CONCLUSION}

We have provided a general understanding of nature fundamental interactions, especially the weak interaction as an interaction between electric and colour charges without assuming a weak charge. The interaction between real energies is the gravitational force, which has three types: mass-mass, mass-radiation, and radiation-radiation interactions.

The interaction between imaginary energies is the electromagnetic (between electric charges), weak (between electric and colour charges), and strong (between colour charges) interactions. In addition, we have four imaginary forces between real and imaginary energies, which are mass-electric charge, radiation-electric charge, mass-colour charge, and radiation-colour charge interactions. Therefore, among the four fundamental elements, we see that we have in total ten (six real and four imaginary) fundamental interactions. In addition, we introduce a three-dimensional energy space to describe all types of matter or particles including quarks and antiquarks [3].

Eqs. (11) and (12) and the issuing discussions show that the gravitational force and the electromagnetic force can be described by exactly the same equation. The only difference here is that in place of electric charge $\mathrm{q}$ we now have the mass $\mathrm{m}$. The different constants $\mathrm{k}$ and $\mathrm{G}$ are simply the indicators of strength of the interacting forces. What is amazing is that the equation describing the interacting force alone is sufficient to derive all the relevant laws governing the electromagnetic and gravitational interactions, including the wave equation. It is a testimony of the consistency of the theory and justification of our generalization of Newton's Law of gravitation to include a dynamic term inferred from the Lorentz force of electrodynamics [12].

The first achievement of our theory is a logical and natural solution to the historical puzzle of action-at-distance. It turns out that the propagation of the gravitational interaction is nothing different from the propagation of electromagnetic wave. There is absolutely no spooky actionat-distance that bothered physicists for over a century. The identity of all the equations that govern the propagation of the interaction is so compelling that we believe the two interactions are propagating through the same universal medium ether, by causing static and dynamic stresses [3].

The second achievement of our theory is the revelation of the essence of the inverse-square law that governs both the electrodynamic and the gravitational interactions. We have demonstrated that the inverse square law is the result of the conservation of the total static and dynamic fluxes as expressed in Gauss' law and the newly discovered Wang's law. The Gauss' Law says that the mass is conserved, and Wang's Law says that the total momentum transmitted into space is conserved [12].

The third achievement of our theory is that it predicted, classically first time ever, that gravitational force is propagated by gravitational wave with the same speed of light. This prediction can be used to test the new generalized theory of gravitation [12].

The unification of gravitational and electromagnetic forces opens new windows for further researches into deeper questions: Why would there be different strengths for the two fundamental interactions? Is charge related to mass? If yes, how? Why do same masses attract while same charges repel? Why there are two types of charges but only one type of mass? The new theory predicts that the speed of gravitation is the same as the speed of light. How to verify 
this prediction experimentally? With high hope for the future, we venture to predict the positive and fruitful results flowing out from this research [12].

\section{REFERENCE}

[1] Boyd, T. J. M., Sanderson, J. J. (2003). The Physics of Plasmas. Cambridge University Press.p.1. ISBN 9780521459129. LCCCN 2002024654

[2] Ball, P. (2004). The Elements: A Very Short Introduction. Very Short Introductions. OUP Oxford.p.33. ISBN 9780191578250

[3] Zhang T.X. Fundamental Elements and Interactions of Nature: A Classical Unification Theory. Progress in Phys., 2010, v.2, 36-42

[4] L. Randall, Warped Passages: Unraveling the Universe's Hidden Dimensions, Ecco, ISBN 0-06-053108-8, 2005.

[5] R. Feynman, F. Morinigo, W. Wagner and B. Hatfield, Feynman lectures on gravitation, Addison-Wesley, ISBN 0-201-62734-5, 1995.

[6] A. Zee, Quantum Field Theory in a Nutshell, Princeton University Press, ISBN 0-69101019-6, 2003.

[7] L. J. Wang, Unification of gravitational and electromagnetic fields, Physics Essays 31(1), 2018.

[8] P. Lorrain and D. Corson, Electromagnetic Fields and Waves, 251, Freeman, San Francisco, 1970.

[9] D. Salisbury and D. Menzel, Nature 252 (1974), 664.

[10] D. W. Sciama and N. Mon, R. Astr. Soc. 113(34), 1953.

[11] L. J. Wang, One hundred years of general relativity - a critical view, Physics Essays 28(4), 2018.

[12] Wang LJ. Classical unification of gravitational and electromagnetic forces (2020) Edelweiss Appli Sci Tech 4: 9-18.

Copyright (C) 2020 The Author(s). This is an Open Access article distributed under the terms of Creative Commons Attribution-NonCommercial-NoDerivatives 4.0 International (CC BYNC-ND 4.0), which permits anyone to share, use, reproduce and redistribute in any medium, provided the original author and source are credited. 